\title{
Comprehensive study of beam focusing by crystal devices
}

\author{
W. Scandale,${ }^{1}$ G. Arduini, ${ }^{1}$ F. Cerutti, ${ }^{1}$ M. Garattini, ${ }^{1}$ S. Gilardoni,${ }^{1}$ A. Masi,${ }^{1}$ D. Mirarchi, ${ }^{1}$ \\ S. Montesano, ${ }^{1}$ S. Petrucci, ${ }^{1}$ S. Redaelli, ${ }^{1}$ R. Rossi, ${ }^{1}$ D. Breton, ${ }^{2}$ L. Burmistrov, ${ }^{2}$ S. Dubos ${ }^{2}$ \\ J. Maalmi ${ }^{2}$ A. Natochii ${ }^{2, \dagger}$ V. Puill, ${ }^{2}$ A. Stocchi, ${ }^{2}$ D. Sukhonos, ${ }^{2, \$}$ E. Bagli,${ }^{3}$ L. Bandiera, ${ }^{3}$ \\ V. Guidi, ${ }^{3}$ A. Mazzolari, ${ }^{3}$ M. Romagnoni, ${ }^{3}$ F. Murtas, ${ }^{1,4}$ F. Addesa, ${ }^{5}$ G. Cavoto, ${ }^{5}$ F. Iacoangeli, ${ }^{5}$ \\ F. Galluccio, ${ }^{6}$ A. G. Afonin, ${ }^{7}$ M. K. Bulgakov, ${ }^{7}$ Yu. A. Chesnokov, ${ }^{7}$ A. A. Durum, ${ }^{7}$ \\ V. A. Maisheev, ${ }^{7, *}$ Yu. E. Sandomirskiy, ${ }^{7}$ A. A. Yanovich, ${ }^{7}$ A. A. Kolomiets, ${ }^{8}$ A. D. Kovalenko, ${ }^{8}$ \\ A. M. Taratin, ${ }^{8}$ G. I. Smirnov, ${ }^{1,8}$ A. S. Denisov, ${ }^{9}$ Yu. A. Gavrikov, ${ }^{9}$ Yu. M. Ivanov, ${ }^{9}$ \\ L. P. Lapina, ${ }^{9}$ L. G. Malyarenko, ${ }^{9}$ V. V. Skorobogatov, ${ }^{9}$ G. Auzinger, ${ }^{10}$ T. James, ${ }^{10}$ \\ G. Hall, ${ }^{10}$ M. Pesaresi, ${ }^{10}$ and M. Raymond ${ }^{10}$ \\ ${ }^{1}$ CERN, European Organization for Nuclear Research, CH-1211 Geneva 23, Switzerland \\ ${ }^{2}$ Laboratore de l'Accelerateur Lineaire (LAL), Université Paris-Sud 11, \\ Centre Scientifique d'Orsay, F-91898 Orsay Cedex, France \\ ${ }^{3}$ INFN Sezione di Ferrara and departamento di Fisica e Scienze della Terra, \\ Universita di Ferrara, Via Sarogat 1 Blocco C, 44121 Ferrara, Italy \\ ${ }^{4}$ INFN, LNF, Via Fermi, 4000044 Frascati (Roma), Italy \\ ${ }^{5}$ INFN Sezione di Roma Roma, Piazzale Aldo Moro 2, 00185 Rome, Italy \\ ${ }^{6}$ INFN Sezione di Napoli, 80126 Napoli, Italy \\ ${ }^{7}$ NRC Kurchatov Institute-IHEP, 142281 Protvino, Russia \\ ${ }^{8}$ Joint Institute for Nuclear Research, Joliot-Curie 6, 141980 Dubna, Russia \\ ${ }^{9}$ Petersburg Nuclear Physics Institute in National Research Centre "Kurchatov Institute", \\ 188300 Gatchina, Russia \\ ${ }^{10}$ Blackett Laboratory, Imperial College, London SW7 2AZ, United Kingdom
}

(Received 30 June 2017; published 18 January 2018)

\begin{abstract}
This paper is devoted to an experimental study of focusing and defocusing positively charged particle beams with the help of specially bent single crystals. Four crystals have been fabricated for this purpose. The studies have been performed at the CERN SPS in $400 \mathrm{GeV} / c$ proton and $180 \mathrm{GeV} / c$ pion beams. The results of measurements of beam envelopes are presented. The rms size of the horizontal profile at the focus was 5-8 times smaller than at the exit of the crystals. The measured focal lengths were 4-21 m. The results of measurements are in good agreement with calculations. Possible applications of focusing crystals in present and future high energy accelerators are discussed.
\end{abstract}

DOI: $10.1103 /$ PhysRevAccelBeams.21.014702

\section{INTRODUCTION}

The possibility to use bent single crystals for deflection of positively charged particle beams proposed and studied in [1] has proven to be very fruitful. In fact, with the help of bent single crystals, the extraction of circulating proton and ion beams has been realized at all the largest modern

*Corresponding author. maisheev@ihep.ru

Ph.D. student at the T. Shevchenko National University of Kyiv.

${ }^{\ddagger}$ Ph.D. student at the T. Shevchenko National University of Kyiv.

Published by the American Physical Society under the terms of the Creative Commons Attribution 4.0 International license. Further distribution of this work must maintain attribution to the author(s) and the published article's title, journal citation, and DOI. circular accelerators [2-5]. In addition, several new phenomena were found and investigated in bent single crystals, such as the rotation of the spin vector of circulating particles [6,7], volume reflection [8-10] and a novel photon radiation process $[11,12]$. Moreover, it has been shown that bent single crystals could be used for focusing beams of positively charged particles [13]. This paper is devoted to further development of focusing crystals and experimental studies of beam focusing devices based on these crystals. The measurements performed in the CERN SPS extracted beams have confirmed improvements in the quality of the focusing crystal device and allowed to develop a stable production technology. New quantitative data and the suppression of aberrations in the focusing process have been obtained.

As is well known, conventional beam optics is based on beam focusing with quadrupole lenses whose fields are not at all comparable in strength with the electrostatic field of a 
bent crystal (the equivalent field is as high as $~ 1000 \mathrm{~T}$ ). The beam focusing devices described in this work have focal lengths from several meters to $100-200 \mathrm{~m}$ and may be used for extraction of secondary particles with energies in the $\mathrm{GeV}$ and $\mathrm{TeV}$ ranges and an angular spread of a few mrad.

The paper is organized as follows. First, we demonstrate progress in manufacture of the focusing crystals; here we present the new samples of focusing crystals used for measurements. Then we give a short description of the experimental setup and describe the procedure of the data analysis. In the following sections we present the experimental results obtained with four crystals in the focusing and defocusing mode, after which a discussion and conclusions follow.

\section{RECENT PROGRESS IN FABRICATION OF FOCUSING CRYSTALS}

The first measurements of the beam focusing effect were performed in the 1990s. The principles of focusing positively charged particle beams and the construction of the focusing crystal device were described in $[13,14]$. To enable focusing, the exit face of a bent crystal is shaped in such a way that the tangents to crystallographic planes at the surface pass through a common line. The bending of the crystal is achieved with the help of two cylindrical metallic surfaces. This massive substance around the crystal significantly complicates the construction providing the necessary bending force, which makes unfeasible the use of crystal deflectors inside the vacuum chamber of circular accelerators.

For the present study, a new technology [15] based on bent strip single crystals [16] was used. The property of anticlastic bending, which accompanies the main primary bending imposed by the applied force, is a common feature of bent strip crystals of a few millimeters length and those of a few centimeters length. The latter have already been employed for beam focusing. Focusing crystals and profile shapes are schematically shown in Fig. 1. The first experiment with the strip focusing crystals [Fig. 1(a)] performed recently is described in [17]. In this experiment, which studied properties of a focusing crystal device, a crystal cut to a triangular shape [triangle BCE in Fig. 1(a)] should have been used. However, a trapezoidal shape was chosen in order to measure different features [2] in a single experiment. Due to this fact, only the upper part of the crystal could be used in the previous measurements. Furthermore, particles moving close to point B [see Fig. 1(a)] are deflected too weakly and hence are mixed with background (nonchanneled) particles.

For the present experiment we have produced crystals which are closer to the ideal shape [see Fig. 1(b)]. The new focusing crystal is now represented by a sum of rectangle $\mathrm{ABCF}$ and triangle FCD. Particles entering the bent crystal are deflected through the same angle over the distance BC (AF). For a sufficiently large deflection angle, the channeled and nonchanneled particles (background) are spatially separated. The triangular part of the crystal deflects particles with different transverse coordinate $x$ according to a linear relationship between the angle and coordinate. Therefore, the particle trajectories converge at some (focal) point.

A photograph of the focusing device and a schematic diagram showing the passage of a beam of particles through the crystal under discussion are displayed in Fig. 2. A high quality polished silicon plate is bent in the vertical direction with the help of a metallic holder. This crystal plate was cut out of silicon along the (111) crystallographic plane and was $2-4 \mathrm{~mm}$ thick and $85 \mathrm{~mm}$ high in the $x$ and $y$ coordinates transverse to the beam direction, respectively. (a)

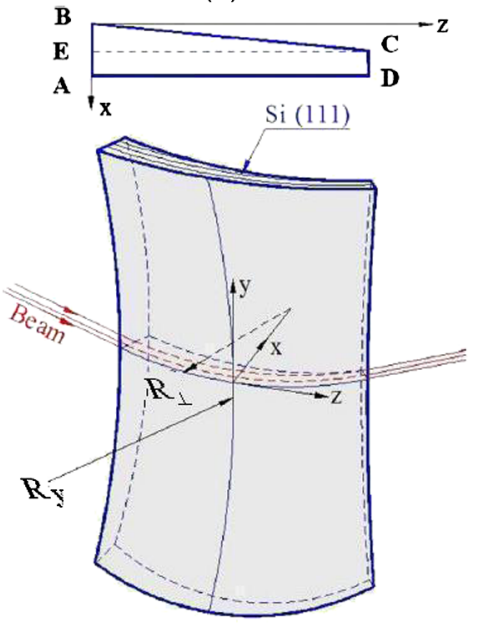

(b)

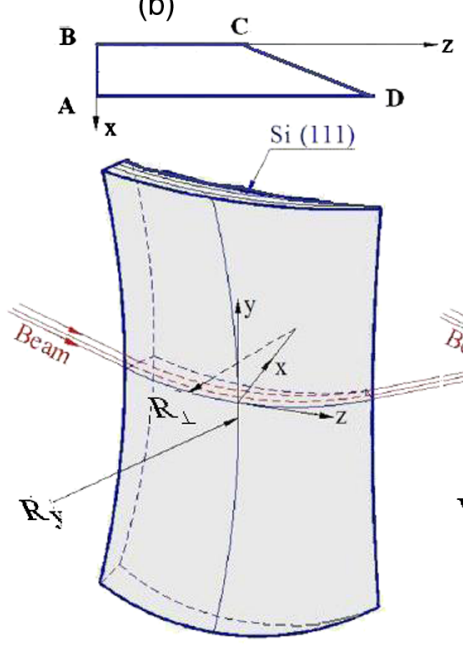

(c)

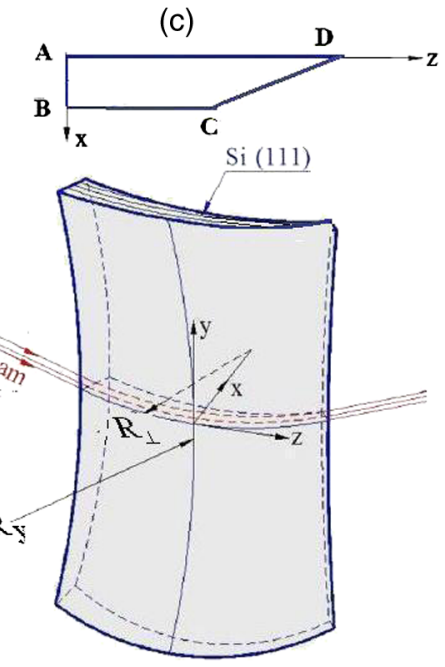

FIG. 1. The bent crystals used for the measurements: (a) focusing crystal in the previous experiment [17], (b) focusing, and (c) defocusing crystals used in the present experiment. $R_{y}$ is the radius of the vertical bending, and $R_{\perp}$ is the radius of the bending in the horizontal plane, which provides the particle beam bending. 


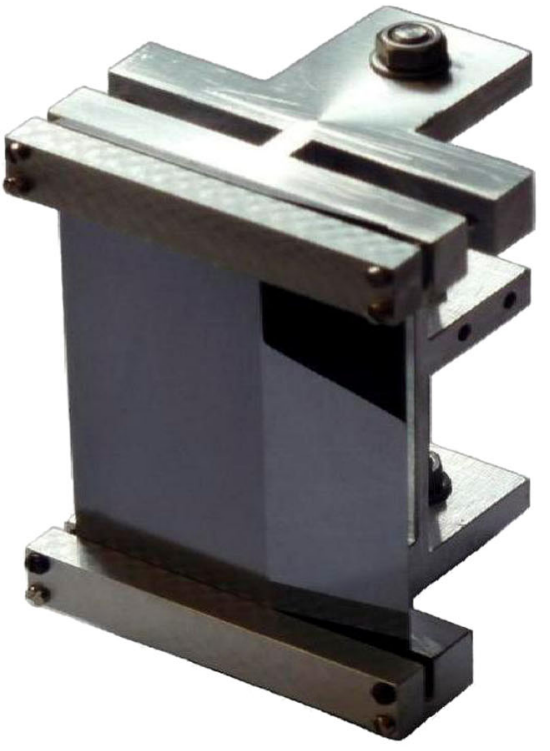

(a)

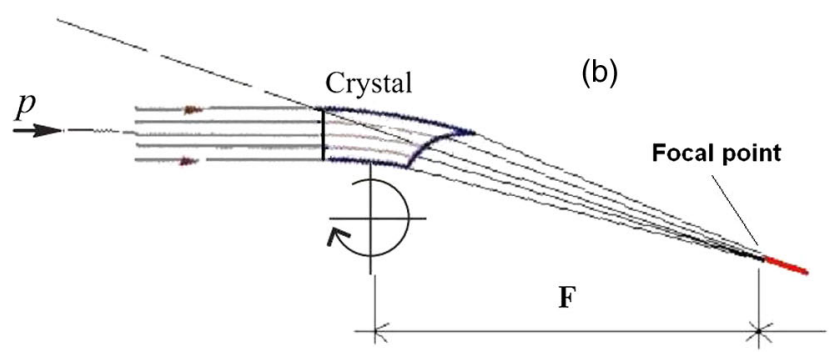

FIG. 2. (a) Photograph of a crystal holder with a focusing Crystal 1; (b) beam focusing scheme using a bent single crystal. For the linear cut, particles accepted in the channeling regime have different bending angles (at different transverse coordinates) and they are focused at some distance from the single crystal.

In the longitudinal $z$ coordinate, the crystal length varied between 30 and $50 \mathrm{~mm}$ owing to the skewed front face. A metal crystal holder created a bending moment in the $y$ coordinate, whereby the crystal was bent transversely with a radius $R$ owing to the anisotropic properties of the crystal lattice (see, e.g., [18] p. 85). This bend was employed to focus and deflect the particles. The focal length $F$ varied from a few meters to $25 \mathrm{~m}$ and the mean deflection angle was a few mrad.

The theoretical description of beam focusing on the basis of bent single crystals can be found in $[15,17,19]$. The focusing crystal can be used also as a beam defocusing device [17]. For this objective, the crystal should be reinstalled in the holder and bent as shown in Fig. 1(c). The strip crystal deflectors described first in Ref. [16] did not have the focusing effect and, therefore, we will refer to them as standard strip crystals.

\section{EXPERIMENTAL SETUP}

The experiment was carried out at the $\mathrm{H} 8$ beam line of the CERN SPS using a practically pure $400 \mathrm{GeV} / c$ proton beam and a $180 \mathrm{GeV} / c$ beam of positive secondary particles for the measurements. The layout of the experiment shown in Fig. 3 was similar to that described earlier in [6]. A high precision goniometer was used to orient the crystal planes with the respect to the beam axis with an accuracy of $2 \mu \mathrm{rad}$. The accuracy of the preliminary crystal alignment with a laser beam was about $0.1 \mathrm{mrad}$. Five pairs of silicon microstrip detectors, two upstream and three downstream of the crystal deflector, were used to measure incoming and outgoing angles of particles with an angular resolution in each arm of about $3 \mu \mathrm{rad}$ [20]. The geometric parameters of the incident beam were measured with the help of the detector telescope. The width of the beam along the horizontal and vertical axes was a few millimeters. The angular divergence of the incident beam in the horizontal and vertical planes was $\sim 10 \mu \mathrm{rad}$ for the proton beam and $\sim 20 \mu \mathrm{rad}$ (horizontal) and $\sim 40 \mu \mathrm{rad}$ (vertical) for the secondary beam of positively charged particles. The average cycle time of the SPS during the measurements was

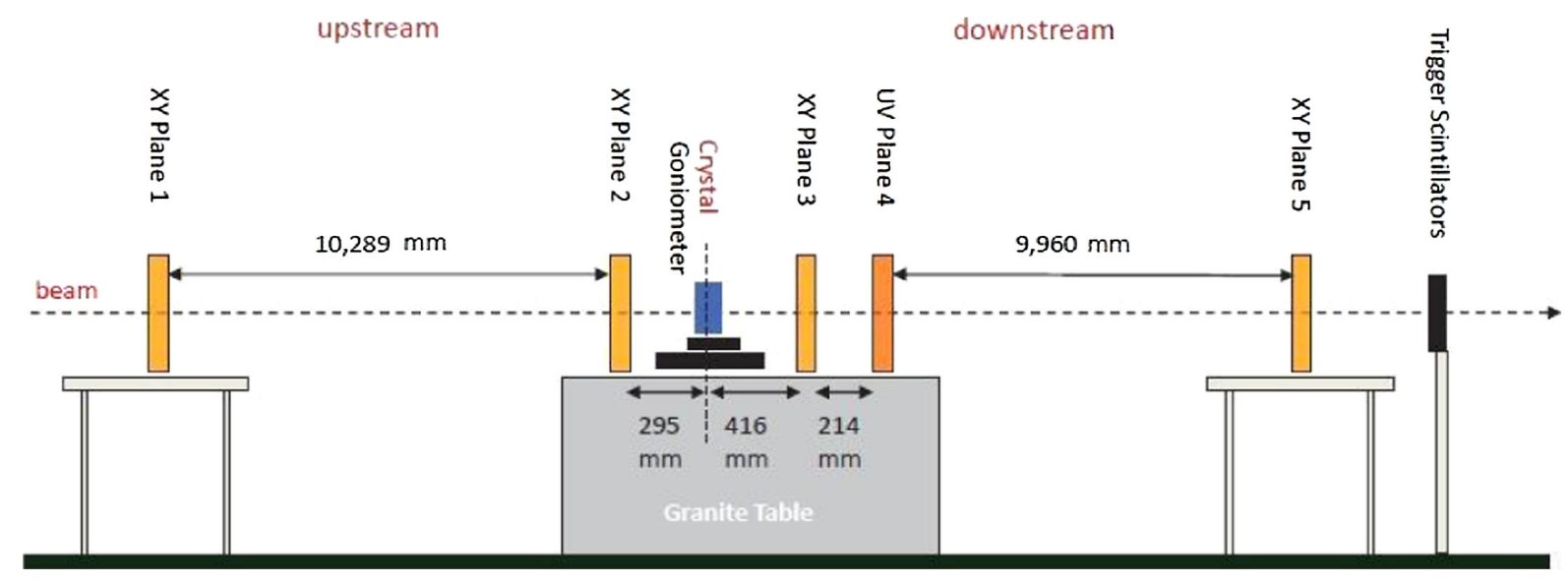

FIG. 3. A schematic view of the layout of the experiment. XY Plane 1-5 are the silicon strip detectors. The bent crystal mounted at the goniometer is installed between strip detectors 2 and 3 . 
about $45 \mathrm{~s}$ with a pulse duration of 10-11 s. Typically, the number of particles per spill was $1.3 \times 10^{6}$.

It should be noted that the system of microstrip detectors in its normal configuration provides measurements only for particle deflection angles smaller than $1.5 \mathrm{mrad}$ because at larger angles deflected particles would miss the last downstream detector.

\section{ANALYSIS OF THE EXPERIMENTAL DATA}

An important task of experimental studies of the beam focusing consists in finding the shape of one-dimensional transverse coordinate particle distributions (in the deflection plane) as a function of the $z$ coordinate along the direction of motion of the beam. Each such distribution can be characterized by rms coordinate values at any $z$ location. Accordingly we can write

$$
s_{x}^{2}(l)=\frac{\sum_{i=1}^{n} x_{i}^{2}(l)}{n}-\left[\frac{\sum_{i=1}^{n} x_{i}(l)}{n}\right]^{2},
$$

where $x_{i}(l)$ is the coordinate of particle (number $i$ ) at a distance $l \geq 0$ from the crystal and $n$ is the total number of particles. Obviously, $x_{i}(l)=x_{i}(0)+\phi_{i} l$, where $\phi_{i}$ is the angular projection of the particle in the deflection plane. Then we obtain

$$
s_{x}^{2}(l)=\left\langle x^{2}\right\rangle-\bar{x}^{2}+\left(\left\langle\phi^{2}\right\rangle-\bar{\phi}^{2}\right) l^{2}+2(\langle x \phi\rangle-\bar{x} \bar{\phi}) l,
$$

where

$$
\begin{aligned}
\left\langle x^{2}\right\rangle & =\frac{\sum_{i=1}^{n} x_{i}^{2}(0)}{n}, \quad \bar{x}=\frac{\sum_{i=1}^{n} x_{i}(0)}{n}, \\
\left\langle\phi^{2}\right\rangle & =\frac{\sum_{i=1}^{n} \phi_{i}^{2}}{n}, \quad \bar{\phi}=\frac{\sum_{i=1}^{n} \phi_{i}}{n}, \\
\langle x \phi\rangle & =\frac{\sum_{i=1}^{n} x_{i}(0) \phi_{i}}{n} .
\end{aligned}
$$

A theoretical description of the beam envelope was presented in $[15,17]$

$$
\begin{aligned}
s_{x}^{2}(l)= & \left\langle x^{2}\right\rangle-\bar{x}^{2}+\left(\left\langle\varphi^{2}\right\rangle-\bar{\varphi}^{2}\right) l^{2}+2(\langle x \varphi\rangle-\bar{x} \bar{\varphi}) l \\
& +\left(\left\langle\theta^{2}\right\rangle-\bar{\theta}^{2}\right) l^{2},
\end{aligned}
$$

where $\left\langle x^{2}\right\rangle$ and $\left\langle\varphi^{2}\right\rangle$ are the mean square size of the beam and the mean square deflection angle at the exit face $(l=0), \bar{x}$ and $\bar{\varphi}$ are the mean size of the beam and the mean deflection angle (for $l=0$ ), $\langle x \varphi\rangle=\int_{0}^{d} x \varphi(x) \rho(x) d x$, and $d$ is the transverse size of a single crystal, which varies in this case from $x_{\min }$ to $x_{\max }$. The function $\rho(x)$ is the distribution (normalized to one) of particles which channeled through the full length of the crystal. The last term $\left(\left\langle\theta^{2}\right\rangle-\bar{\theta}^{2}\right) l^{2}$ is due to variations in the particle exit angle (for those traversing the crystal in the channeling regime). If the distribution of particles over the angle $\theta$ is known we can calculate this last term.

Equation (6) was written for the general case. We can apply this equation for our particular case when the cut is a linear function. In this case, the deflection angle is equal to:

$$
\varphi(x)=\frac{z(x)}{R}=\frac{z_{0}+k x}{R},
$$

where $z_{0}=B C=A F, R$ is the bending radius of a crystal deflector and $k$ is equal to the tangent of angle FCD [see Fig. 1(b)]. Then we obtain

$$
s_{x}^{2}(l)=\left(\left\langle x^{2}\right\rangle-\bar{x}^{2}\right)\left(1+\frac{k l}{R}\right)^{2}+\left(\left\langle\theta^{2}\right\rangle-\bar{\theta}^{2}\right) l^{2} .
$$

From these relations we can find the focal length

$$
L_{f}=-\frac{R / k}{1+q}
$$

and the rms value of the coordinate at the focal point

$$
s_{x}^{2}\left(L_{f}\right)=\frac{\left(\left\langle\theta^{2}\right\rangle-\bar{\theta}^{2}\right) R^{2} / k^{2}}{1+q}=\frac{\left(\left\langle x^{2}\right\rangle-\bar{x}^{2}\right) q}{1+q},
$$

where

$$
q=\frac{\left\langle\theta^{2}\right\rangle-\bar{\theta}^{2}}{\left\langle x^{2}\right\rangle-\bar{x}^{2}} \frac{R^{2}}{k^{2}} .
$$

Below we will demonstrate that $q \ll 1$ for all beam deflectors based on silicon crystals investigated in the present study.

Thus, from Eq. (8) we see that the beam envelope is a quadratic function of the longitudinal coordinate $l$ depending on the three parameters: $\left\langle x^{2}\right\rangle-\bar{x}^{2}, k / R$ and $\left\langle\theta^{2}\right\rangle-\bar{\theta}^{2}$. Note that $L_{f}>0$ if $k<0$ and this case corresponds to a beam focusing crystal. The opposite case, $k>0$, corresponds to a defocusing crystal. The case $k=0$ describes the standard strip crystal.

\section{ACCEPTANCE OF BEAM PARTICLES INTO THE CHANNELING REGIME}

Particles incident on the crystal at a small angle (less than the critical angle for channeling, $\theta_{c}$ ) relative to the (111) crystallographic plane have a nonzero probability to be captured into the channeling regime. The particles which were accepted into the channeling regime at the entrance of a crystal deflector can be focused (defocused) with the help of devices shown in Fig. 1. In the present experiment, we studied the focusing (defocusing) of a parallel beam into a pointlike image (into a wide divergent beam) in the bending plane. 
The total capture probability for the (111) plane in a silicon crystal and large bending radii was defined in the following way. As is known, the (111) plane consists of wide and narrow planes. The potential barrier $U_{0}$ equals $22.5 \mathrm{eV}$ and $7.6 \mathrm{eV}$ [21] for the wide and narrow planes, respectively. To capture particles into the channeling regime, it is necessary to fulfill the condition: $E_{0} \theta^{2} / 2+U(x) \leq U_{0}$, where $E_{0}$ is the particle energy, $U(x)$ is the potential function, and $\theta \leq \theta_{c}$ is the incident particle angle with respect to the $z$ axis. Taking into account this equation and assuming a uniform distribution of particles within the channeling angle $\left( \pm \theta_{c}\right)$ it is easy to show that $\approx 61 \%$ and $\approx 10 \%$ of the beam will be captured into channeling in the wide and narrow planes, respectively. However, for the narrow plane the dechanneling length is small, and for sufficiently long crystals a fraction of particles arriving in narrow channels will be lost due to dechanneling. Knowing the dechanneling length for the wide plane $l_{d}$ (see [18]) we can estimate the fraction of the $61 \%$ of particles, which exit from the deflector in the channeling regime to be $0.61 \exp \left(-L / l_{d}\right)=0.53$. Here $l_{d}=30.8 \mathrm{~cm}$ for $E_{0}=400 \mathrm{GeV}$ and $L=4 \mathrm{~cm}$ is the mean thickness of the silicon deflector along the $z$ axis. This is only an estimate; a more precise calculation should take into account such features of particle propagation over a short depth in the crystal as nuclear dechanneling [22] and include some correction to the critical angles for channeling due to crystal bending. Thus we believe that a more precise calculation would slightly decrease our estimate.

As a result, we expect that the relative number of particles channeled through the full length of the crystal deflector will be slightly less than $50 \%$ of the initial total number within the $\pm \theta_{c}$ angular range.

Fig. 4 shows a two-dimensional plot of the distribution of events as a function of the transverse horizontal coordinate and the horizontal deflection angle, respectively. In this figure, the results of measurements for the focusing Crystal 1 and $400 \mathrm{GeV} / c$ beam momentum are presented. In the upper part of the plot, one can see the intensity distribution of nonchanneled particles. The area between letters $A_{ \pm}$and $B_{ \pm}$(in the horizontal direction) and between straight lines $A_{-} B_{-}$and $A_{+} B_{+}$(in the vertical direction) corresponds to particles which were scattered on the crystal material but were not captured into the channeling regime. The straight line $O O^{\prime}$ shows the angular center of the exit of the channeled particles from the crystal deflector. A clear linear dependence of the deflection angle of emerging particles on the coordinate is observed. The conclusion is based on consideration of the channeled particles within the straight line segment from -0.94 to $1.01 \mathrm{~mm}$ of the abscissa in Fig. 4.

The angular distribution of particles detected downstream of the beam focusing device is shown in Fig. 5. One can clearly see three characteristic regions of the interacting particles.

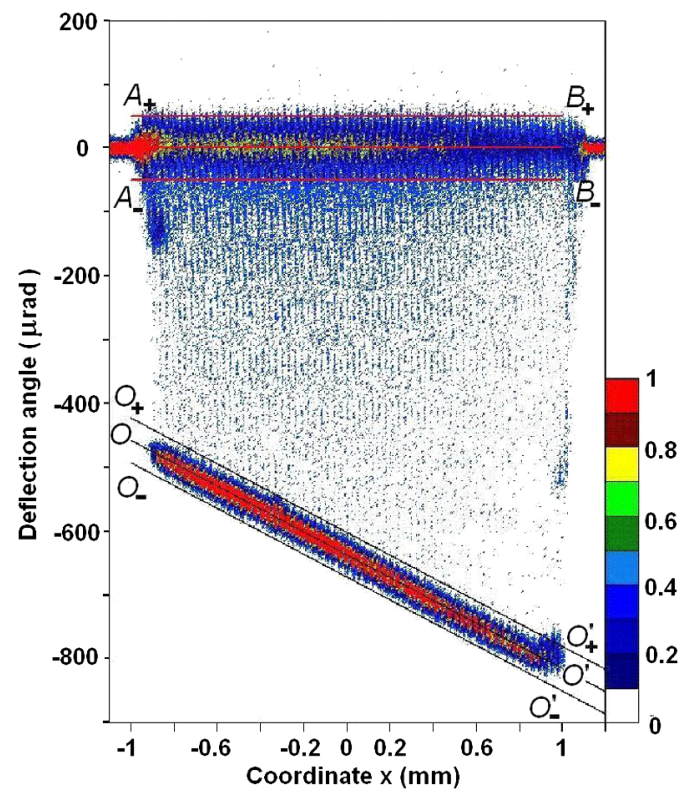

FIG. 4. Beam intensity recorded by the microstrip detectors as a function of horizontal coordinate $x$ and horizontal deflection angle. The maximum of the intensity distribution is normalized to 1 (color band). The area between lines $A_{+} B_{+}$and $A_{-} B_{-}$ corresponds to nonchanneled particles, which pass through the crystal after scattering. Because of the difference of the paths of nonchanneled particles, the particle density decreases from left to right along $A_{+} B_{ \pm}$lines due to multiple Coulomb scattering. The line $O O^{\prime}$ corresponds to the center of the distribution of channeled particles which passed along the full crystal length. The particles between $A_{-} B_{-}$and $O_{+} O_{+}^{\prime}$ correspond to the dechanneled beam fraction. The beam momentum is $400 \mathrm{GeV} / c$.

Between the two regions of emerging channeled and nonchanneled events, a region of dechanneled events is located, where particles entering the crystal were captured into the channeling regime but later dechanneled before

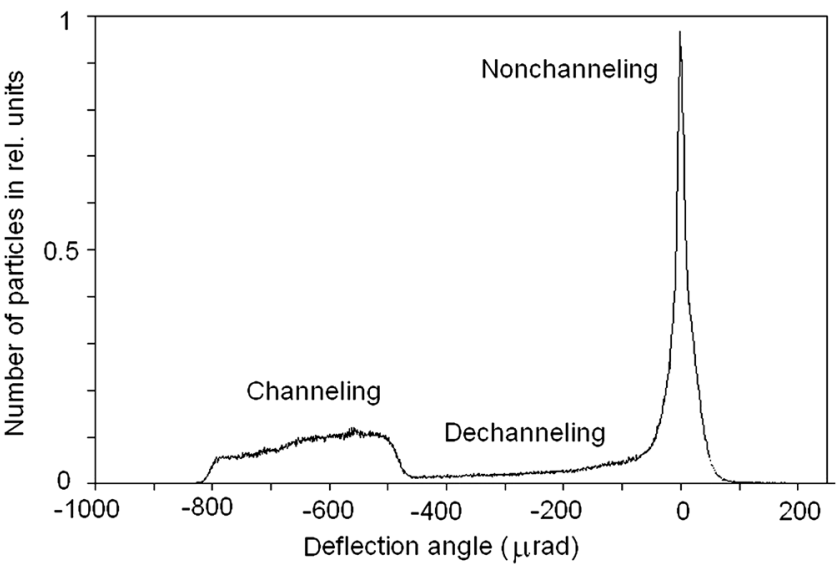

FIG. 5. The angular distribution of particles which passed through the focusing crystal. Three characteristic regions are visible: nonchanneling, channeling, and dechanneling. The histogram corresponds to the projection of the $2 \mathrm{D}$ distribution in Fig. 4 within the interval $-0.94 \mathrm{~mm}<x<1.01 \mathrm{~mm}$. 
they could reach the exit of the crystal deflector. The straight line $O O^{\prime}$ can be approximated with the following relation:

$$
\theta(x)=p_{1} x+p_{0},
$$

where the coefficients $p_{1}=-178.8 \pm 0.06 \mu \mathrm{rad} / \mathrm{mm}$ and $p_{0}=-636.5 \pm 0.03 \mu \mathrm{rad}$. The particles, which were captured into the channeling regime at the entrance to the deflector and pass through the crystal to the exit, lie between the two curves $O_{+} O_{+}^{\prime}=\theta(x)+\theta_{0}$ and $O_{-} O_{-}^{\prime}=$ $\theta(x)-\theta_{0}$. The analysis described in the following section demonstrated that $\theta_{0}$ can be estimated as $\theta_{0}=3 \theta_{c}$.

There are several factors, which determine the size of the area between curves $O_{+} O_{+}^{\prime}=\theta(x)+\theta_{0}$ and $O_{-} O_{-}^{\prime}=$ $\theta(x)-\theta_{0}$ by contributing to the angular spread due to (1) channeling: the mean square variation of this value is estimated to be $\theta_{c}^{2} / 3$; (2) multiple Coulomb scattering in material after the crystal (two microstrip detectors and beam pipe elements); (3) measurement uncertainty; (4) imperfections of the entrance and exit surfaces of the crystal.

For the incident proton energy $E_{0}=400 \mathrm{GeV}, \theta_{c} \approx$ $10.6 \mu \mathrm{rad}$. For sufficiently large bending radii, the critical angle $\theta_{c}^{*}(R) \approx \theta_{c}$. For example, for $R=55 \mathrm{~m}, \theta_{c}^{*} \approx 10.4 \mu \mathrm{rad}$.

Let us assume that a divergent proton beam passes through the bent crystal. In the data analysis, we have found the direction which coincides with the direction of the (111) crystallographic plane. This direction corresponds to the maximum flux of protons captured in the channeling regime. It is clear that an ideal crystal deflector can capture particles within the range $\pm \theta_{c}^{*}(R)$. The range boundaries are however shifted due to the angular measurement uncertainty. Therefore, we estimate the experimental limits as $\pm 12.5 \mu \mathrm{rad}$.

The number of channeled particles $N_{c h}$, which passed through the entire crystal deflector and corresponds to the angular range of $\pm \psi$ is shown in Fig. 6 as a function of the maximum entrance angle $\psi$, by curve $A$, after normalization to the total number of particles in the area between the $O_{-} O_{-}^{\prime}$ and $O_{+} O_{+}^{\prime}$ lines in Fig. 4. This number becomes larger than 0.9 for entrance angles larger than $\sim 12.5 \mu \mathrm{rad}$, which means that more than $90 \%$ of particles with the noted properties have an entrance angle less than $12.5 \mu \mathrm{rad}$ as we expected.

The ratio of the number of channeled particles $N_{c h}$ to the total flux measured within an angular range $\pm \psi$ is shown in Fig. 6 by curve $B$. The ratio corresponds to the efficiency of finding a focused beam particle, and it is $\approx 40 \%$ of the total particle flux.

In addition, one can conclude from the shape of curve $A$ that the crystallographic structure of the silicon deflector did not deteriorate after its fabrication and bending. Otherwise, the number of channeled particles with the



FIG. 6. The measured angular characteristics in the bending plane. $N_{\mathrm{Ch}} / N_{\mathrm{Ch} \text {, max }}$ (curve $A$ ) is the number of channeled particles as a function of the maximum entrance angle $\psi$ normalized to the total number of channeled particles $N_{\mathrm{Ch} \text {,max }}$, which is constant for $\psi \geq 20 \mu \mathrm{rad}$. On the right ordinate axis, $\varepsilon$ (curve $B$ ) is the ratio of the number of channeled particles to the total number of particles within the maximum entrance angle. Data from $-0.94 \mathrm{~mm}<x<1.01 \mathrm{~mm}$ are included (see Fig. 4).

entrance angles larger than $\sim 12.5 \mu \mathrm{rad}$ would be lower than 0.9 .

\section{RESULTS OF THE BEAM FOCUSING STUDIES}

Here we present the results of the data analysis for the focusing Crystal 1. These results proved to be in agreement with the equations of Sec. IV. First we found the beam envelope downstream of the crystal deflector using Eqs. (1)-(5). These equations are based on the definition of the beam envelope and hence have a common character. Then we calculated the envelope with the help of Eq. (8), which was obtained for the special case of a linear cut of the silicon crystal. As explained earlier, according to Eq. (8), the beam envelope is represented by a quadratic curve as a function of three parameters: $\left\langle x^{2}\right\rangle-\bar{x}^{2}, k / R$, and $\left\langle\theta^{2}\right\rangle-\bar{\theta}^{2}$. These parameters can be found from the experimental data. In fact, $k / R=p_{1}$ is calculated from a comparison of the theoretical dependence [see Eq. (7)] with the measured dependence in Eq. (12). The properties of the value of $\left\langle\theta^{2}\right\rangle-\bar{\theta}^{2}$ are discussed in Section V after Eq. (12). The measured distribution of particles over $\theta$ is shown in Fig. 7.

The results of reconstruction of the beam envelope of the $400 \mathrm{GeV} / c$ proton beam are shown in Fig. 8. To this end, we used the two methods described in Section IV. The first method [see Eqs. (1)-(5)] has a general character and it may be applied for analysis of any beam and focusing crystal. The second method [see Eq. (8)] may be applied only for the focusing crystal with a linear cut. In practice, the envelopes reconstructed with the help of these two methods do not differ very much. The focal lengths obtained are 


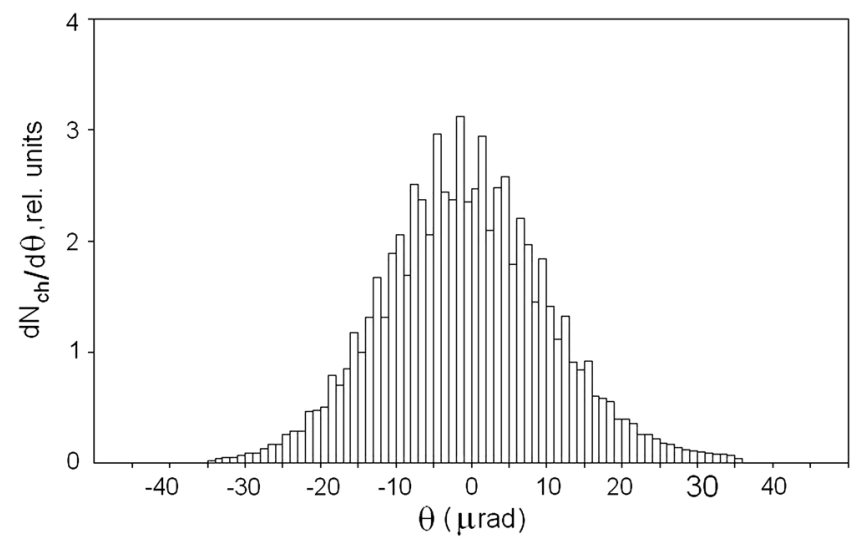

FIG. 7. The distribution of $400 \mathrm{GeV} / c$ beam protons as a function of the angle $\theta$.

$5.503 \mathrm{~m}$ and $5.509 \mathrm{~m}$ for the first and second methods, respectively. The rms size of the focal points is found to be $0.060 \mathrm{~mm}$ and $0.0602 \mathrm{~mm}$, respectively.

The various measured beam profiles are shown at the bottom of Fig. 8. In particular, the initial beam profile (with rms size $0.492 \mathrm{~mm}$ ) is presented here. It shows that the size of the focused beam is about 8.2 times smaller than the initial one (at the exit from the crystal deflector). For definiteness, $\left\langle x^{2}\right\rangle-\bar{x}^{2}=0.242 \mathrm{~mm}^{2}$, $\left\langle\phi^{2}\right\rangle-\bar{\phi}^{2}=7885.35 \mu \mathrm{rad}^{2}, 2(\langle x \phi\rangle-\bar{x} \bar{\phi})=86.78 \mathrm{~mm} \cdot \mu \mathrm{rad}$, $k / R=178.8 \mu \mathrm{rad} / \mathrm{mm}$, and $\left\langle\theta^{2}\right\rangle-\bar{\theta}^{2}=117.44 \mu \mathrm{rad}^{2}$.

The results presented here were obtained for an entrance angle $\psi$ approximately equal to the channeling critical angle $\theta_{c}$. We observed a very small difference between beam envelopes for different angles $\psi$. The prediction made for the ideal case, when the proton beam propagates in vacuum downstream of the crystal deflector with perfect crystallographic structure, is shown by a thin solid line in Fig. 8.

The effect of beam defocusing was investigated in the $180 \mathrm{GeV} / c$ secondary particle beam of the CERN SPS using Crystal 1 reinstalled in a holder as shown in Fig. 1(c). Figure 9 is similar to Fig. 4 and corresponds to the measurements in a $180 \mathrm{GeV} / c$ beam, and to the defocusing mode for Crystal 1. We describe the center of the distribution of channeled particles with the help of a linear function [see Eq. (12)]. For the defocusing crystal, $p_{1}>0$ as we expected.

The reconstructed envelope of the $180 \mathrm{GeV} / c$ secondary beam downstream of the defocusing device is shown in Fig. 10. Note that the rms beam size is about five times larger at a distance of $11 \mathrm{~m}$. As in the previous case, we used two methods of obtaining envelopes which yielded practically the same results. We obtained the following values for the main parameters: $\left\langle x^{2}\right\rangle-\bar{x}^{2}=0.137 \mathrm{~mm}^{2}$, $\left\langle\phi^{2}\right\rangle-\bar{\phi}^{2}=18822.8 \mu \mathrm{rad}^{2}, 2(\langle x \phi\rangle-\bar{x} \bar{\phi})=100.39 \mathrm{~mm} \cdot \mu \mathrm{rad}$, $k / R=368.5 \mu \mathrm{rad} / \mathrm{mm}$, and $\left\langle\theta^{2}\right\rangle-\bar{\theta}^{2}=439.18 \mu \mathrm{rad}^{2}$.

The plot in Fig. 10 shows that the initial coordinate distribution of the beam and mean angle of deflection differ

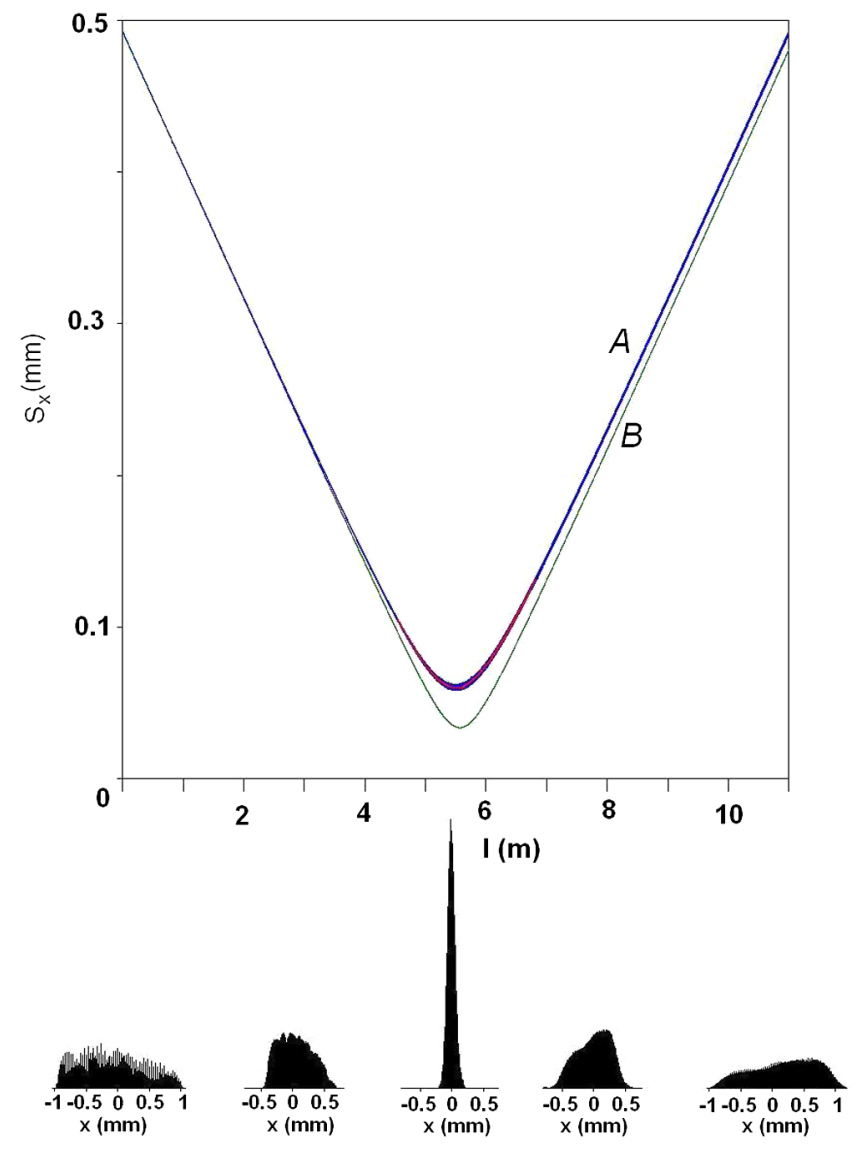

FIG. 8. Top panel: results for the envelope reconstruction of the $400 \mathrm{GeV} / c$ proton beam as a function of distance $l$ from the crystal deflector. The two lines labeled $A$ are calculated using Eqs. (1) and (8) (blue and red lines, respectively), and they practically coincide. The width of the blue line corresponds to measurement uncertainties [20]. Near the focal point, the uncertainty is the largest and is equal to $\approx 0.0025 \mathrm{~mm}$. Line $B$ is a prediction for the case when the beam propagates in vacuum downstream of the crystal. Bottom panel: the beam profiles (from left to right) for $l=0, L_{f} / 2, L_{f}, 3 L_{f} / 2,2 L_{f}$, respectively.

from the data corresponding to the case of using the crystal in the focusing mode. The change of the parameter $\left\langle x^{2}\right\rangle-\bar{x}^{2}$ is due to stronger dechanneling at a beam momentum of $180 \mathrm{GeV} / c$ and a different crystal orientation with respect to the beam.

Curve $B$ in Fig. 10 shows the calculated beam envelope for the standard strip crystal. In the calculation, Eq. (8) with the parameter $k=0$ has been used.

It is noteworthy that the results of the measured characteristics represented by Eqs. (3)-(5) for the focusing mode of a crystal deflector allow one to find the beam envelope for the same crystal but used in the defocusing mode. To this end, we should change the sign of the term $(\langle x \phi\rangle-\bar{x} \bar{\phi})$ in Eq. (6). Analogously, Eq. (8) can be used for calculation of the beam envelope in the defocusing mode.

Based on the measurements for the focusing device, for a $400 \mathrm{GeV} / c$ proton beam we can obtain the beam envelope 


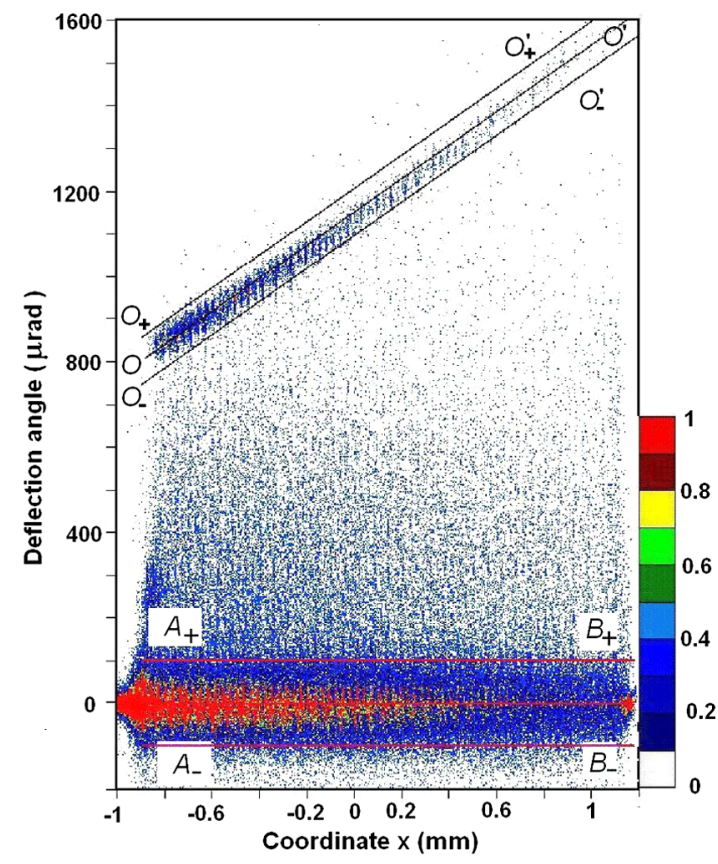

FIG. 9. Beam intensity recorded by the microstrip detectors as a function of horizontal coordinate $x$ and horizontal deflection angle. The area between lines $A_{+} B_{+}$and $A_{-} B_{-}$corresponds to nonchanneled particles which pass through the crystal after they were scattered. The line $O O^{\prime}$ corresponds to the center of the distribution of channeled particles, which passed along the full crystal length. The particles between $A_{+} B_{+}$and $O_{-} O_{-}^{\prime}$ correspond to the dechanneled fraction. The beam momentum is $180 \mathrm{GeV} / c$.

for the case of a defocusing crystal deflector shown by curve $C$ in Fig. 10.

In a similar manner, we made measurements of focusing characteristics for Crystals 2, 3, and 4. The first two crystals

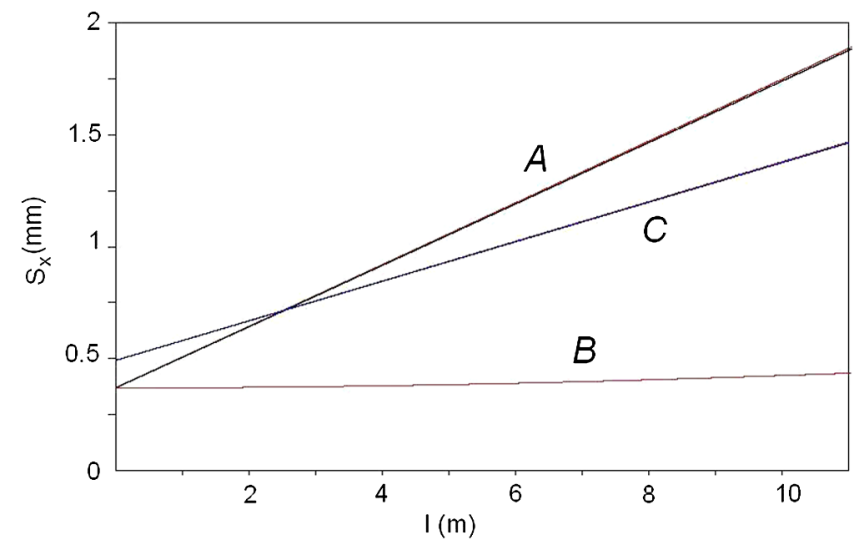

FIG. 10. Reconstructed beam envelope for a $180 \mathrm{GeV} / c$ secondary beam (line $A$ ) as a function of distance $l$ from the Crystal 1, which was transformed into the defocusing one. The calculated uncertainty of the measured envelope function does not exceed $0.001 \mathrm{~mm}$. Line $B$ is the calculated beam envelope downstream of the standard strip crystal, and line $C$ is the prediction of the beam envelope for a $400 \mathrm{GeV} / c$ proton beam for the defocusing case. have sizes (width, height, and thickness) very similar to Crystal 1. Crystal 2 was positioned in the defocusing mode and Crystal 3 was in the focusing mode. Crystal 4 has a width of $4 \mathrm{~mm}$ and was used in the focusing mode. The study of all these crystals was performed in the $180 \mathrm{GeV} / c$ secondary beam; parameter $|k| / R$ was found experimentally for each crystal.

We have reconstructed the beam envelope for all four crystals. For Crystal 2, the beam envelope is similar to that of Crystal 1 (in the defocusing mode, see Fig. 8) and represents practically a straight line with an rms coordinate size $\approx 0.5 \mathrm{~mm}$ at $l=0$ and $\approx 2.1 \mathrm{~mm}$ at $l=11 \mathrm{~m}$. For Crystal 3, the focal point is at $l=4.30 \mathrm{~m}$ and the initial beam size is decreased by a factor 5.2 at the focus. As with Crystal 1, we obtained very similar results from reconstruction of the envelope for Crystals 2 and 3, using both methods.

Crystal deflector 4 has a bending radius of about $200 \mathrm{~m}$ and we expected a large focal length for this crystal, $R / k \sim 20 \mathrm{~m}$. The results of envelope reconstruction are displayed in Fig. 11. Note that the focal length is about $21 \mathrm{~m}$, and only a small difference between the two reconstruction methods is observed.

The main characteristics of focusing devices measured in the experiment are presented in Table I. Crystal 4 has $4 \mathrm{~mm}$ transverse thickness (in the bending plane) and other crystals have a thickness $\approx 2 \mathrm{~mm}$. In the table, positive focal length corresponds to focusing crystals and negative values correspond to defocusing crystals.

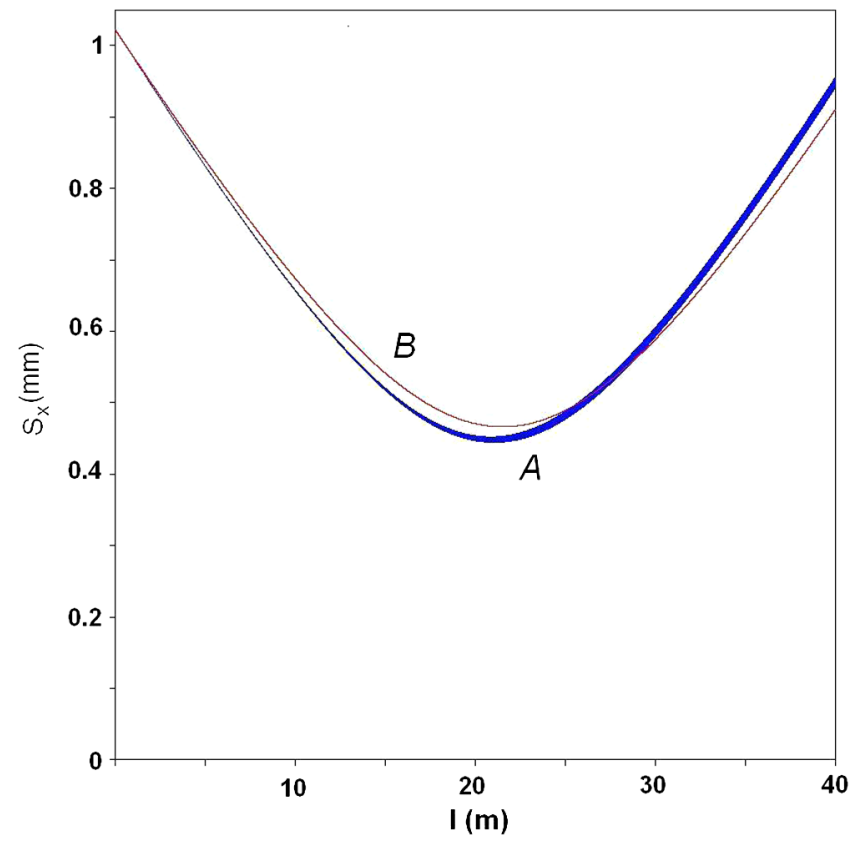

FIG. 11. Reconstructed beam envelope for the focusing Crystal 4 . The width of the line $A$ corresponds to the measurement uncertainties [20] Lines $A$ and $B$ were calculated using Eqs. (1) and (8), respectively. 
TABLE I. The measured characteristics of focusing (defocusing) crystal deflectors. $N$ is the crystal number, $L_{f}, s_{x}\left(L_{f}\right)$ and $L_{f}^{*} s_{x}^{*}\left(L_{f}^{*}\right)$ are the focal lengths and rms sizes (at the focal point), which were obtained with the help of Eqs. (1)-(5) and Eqs. (8)-(11), respectively, $s_{x}(0)$ is the initial rms size at $l=0, F=s_{x}(0) / s_{x}^{*}\left(L_{f}^{*}\right), \theta_{1}=\left(\left\langle\theta^{2}\right\rangle-\bar{\theta}^{2}\right)^{1 / 2}$ and $q$ is defined by Eq. (11). The focal lengths and rms sizes are measured in $\mathrm{m}$ and $\mathrm{mm}$ respectively, angle $\theta_{1}$ is measured in $\mu \mathrm{rad}$, and $k / R$ is measured in $\mu \mathrm{rad} / \mathrm{mm}$.

\begin{tabular}{lrrlllllll}
\hline \hline$N$ & \multicolumn{1}{c}{$L_{f}$} & \multicolumn{1}{c}{$L_{f}^{*}$} & $s_{x}(0)$ & $s_{x}\left(L_{f}\right)$ & $s_{x}^{*}\left(L_{f}^{*}\right)$ & \multicolumn{1}{c}{$F$} & $\theta_{1}$ & $k / R$ \\
\hline 1 & 5.503 & 5.509 & 0.492 & 0.0600 & 0.0602 & 8.20 & 10.84 & 178.8 \\
1 & -2.667 & -2.651 & 0.370 & 0.0561 & 0.0562 & 6.58 & 20.96 & 368.5 & 0.0152 \\
2 & -3.650 & -3.621 & 0.5232 & 0.0737 & 0.0733 & 7.098 & 20.04 & 270.7 & 0.02 \\
3 & 4.292 & 4.341 & 0.452 & 0.088 & 0.089 & 5.16 & 20.07 & 221.4 & 0.0403 \\
4 & 20.96 & 21.52 & 1.02 & 0.448 & 0.466 & 2.28 & 19.28 & 36.8 & 0.263 \\
\hline \hline
\end{tabular}

The rms beam size at the focal point was found with the help of the coupling between the characteristics of focusing and defocusing crystals. The coupling between beam envelopes follows from Eqs. (7)-(10). The function relating the envelopes $s_{x}$ for the cases of focusing and defocusing depends on $l$ and $l_{d}$, which are the distances downstream of the focusing and defocusing crystal, respectively. This allows one to evaluate the envelope for the defocusing case using the known beam envelope for the focusing crystal as $s_{x}\left(l_{d}\right)=s_{x}\left(l-2 L_{f}\right)$. The beam envelope for the two cases is displayed in Fig. 12 as a single function, which is done by shifting the origin for the defocusing case to $l=11 \mathrm{~m}$. In other words, the envelopes coincide if $l \geq 2 L_{f}$

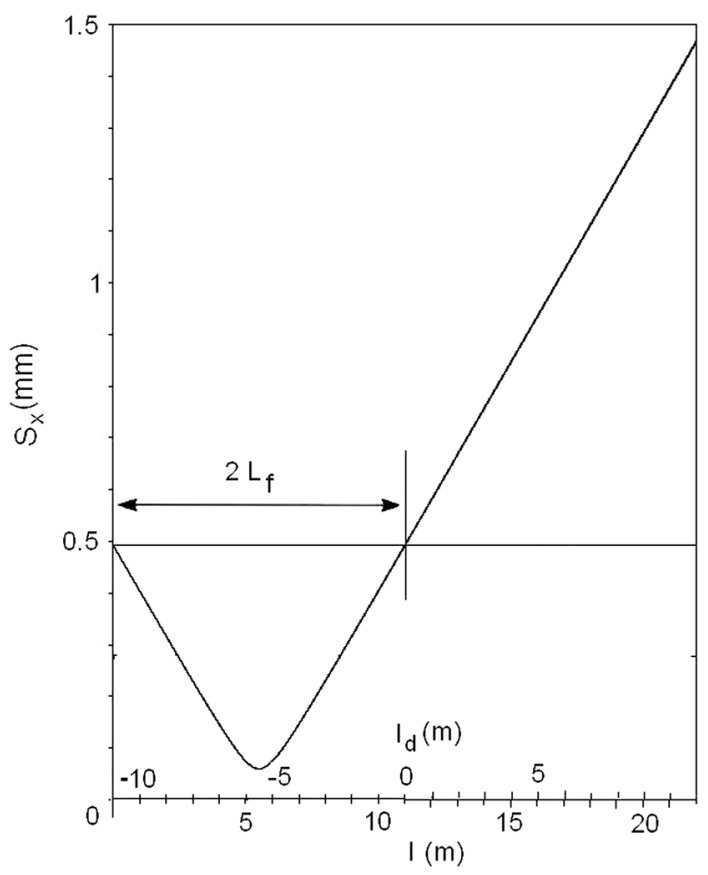

FIG. 12. The universal function describing the envelope for the focusing and defocusing case obtained from the experimental study of a focusing crystal in a $400 \mathrm{GeV} / c$ proton beam. The values for the distance $l$ from the focusing and $l_{d}$ from the defocusing crystal are shown on the abscissa axis with tick marks pointing upwards and downwards, respectively. The thin horizontal line corresponds to the initial rms size of the beam of $0.492 \mathrm{~mm}$ at $l=0$ or $l_{d}=0$. or $l_{d} \geq 0$. The thin horizontal line corresponds to the initial rms size of the beam of $0.492 \mathrm{~mm}$ at $l=0$ or $l_{d}=0$.

In practice, the transition to the defocusing case is done by replacing the crystal shown in Fig. 1(b) with the one shown in Fig. 1(c). For this action to be successful, it is necessary that the characteristic parameters $s_{x}(0), \theta_{1}, R / k$ are conserved.

\section{DISCUSSION}

The goal of the present study was a detailed analysis of one of the applications of a bent single crystal, namely focusing of a parallel beam of high energy positive particles to a pointlike image. As we have shown in Sec. VI, the same design of a crystal-based device can transform a parallel particle beam into a substantially divergent beam. An alternative option is to use a crystal-based device for transformation of a narrow beam with high divergence reaching $\sim 10^{3} \theta_{c}$ into a parallel beam in the bending plane. In this scenario, the beam emerging from the target downstream of the crystal deflector placed at a distance $l=L_{f}$ from the target is incident onto the crystal similar to what is shown in Fig. 2(b) rotated in the holder by $180^{\circ}$ with respect to the normal to the bending plane. This possibility of handling the charged particle beam was considered theoretically and experimentally in Refs. [14,23,24].

To make the usage of focusing and defocusing crystals more straightforward, a number of fabrication problems should be solved and an adequate mathematical description of the crystal properties is needed. This is why these questions have been studied in detail in the present experiment. The data analysis has demonstrated that the predictions made for the shape of the beam envelope with a three parameter quadratic function is valid with high accuracy if parameters $\left\langle x^{2}\right\rangle-\bar{x}^{2}, k / R$ and $\left\langle\theta^{2}\right\rangle-\bar{\theta}^{2}$ are used. Important characteristics such as the focal length and size of the beam at the focal point are expressed using these parameters [see Eqs. (9)-(10)]. Note, that the value of $q$ in these equations is small and lies in the range from 0.015 to 0.26 for Crystal $1\left(E_{0}=400 \mathrm{GeV}\right)$ and Crystal 4 $\left(E_{0}=180 \mathrm{GeV}\right)$, respectively.

It is important to understand the nature of these parameters as well as their impact on focusing characteristics. For 
the mean square positional value, one can assume $d^{2} / 12$ as a first approximation, where $d$ is the width of the crystal along $x$ direction. The assumption corresponds to a uniform distribution of the beam particles. In reality, this parameter depends on the dechanneling process and, in principle, it can be taken into account in preliminary calculations.

The physical meaning of $k / R$ is also clear. The value $R / k$ is the approximate focal length of a beam focusing device, and this value can be found with good accuracy in laboratory conditions before use in experiments.

The parameter $\left\langle\theta^{2}\right\rangle-\bar{\theta}^{2}$ is the least predictable. The possible nature of this parameter was discussed in Sec. V, prior to Eq. (12). In the case of beam propagation in vacuum downstream of the beam focusing device, this parameter will be determined only by the mean square exit angle. If we assume a uniform angular distribution of particles within the critical channeling angle $\theta_{c}$, we obtain $\left\langle\theta^{2}\right\rangle-\bar{\theta}^{2} \approx \theta_{c}^{2} / 3$. For the $400 \mathrm{GeV} / c$ and $180 \mathrm{GeV} / c$ beams, $\theta_{c}^{2} / 3 \approx 37.5 \mu \mathrm{rad}^{2}$ and $83.3 \mu \mathrm{rad}^{2}$, respectively. The measured value of $\left\langle\theta^{2}\right\rangle-\bar{\theta}^{2}$ was $117.5 \mu \mathrm{rad}^{2}$ at $400 \mathrm{GeV} / c$ and about $400 \mu \mathrm{rad}^{2}$ at $180 \mathrm{GeV} / c$. This difference can be explained by the fact that part of the contribution has a linear behavior (depending on $\theta_{c}$ ) while the multiple scattering contribution has a quadratic behavior in amorphous media. From Eqs. (10) and (11) the reduction factor is

$$
\sqrt{\frac{\left(\left\langle x^{2}\right\rangle-\bar{x}^{2}\right)}{s_{x}^{2}\left(L_{F}\right)}}=\sqrt{1+1 / q} .
$$

These considerations show that the factor increases as $\sqrt{E_{0}}$ or faster. Therefore, for Crystal 1, the reduction factor at $E_{0}=6500 \mathrm{GeV}$ will be $\approx 32$, and the rms beam size will be $\approx 0.015 \mathrm{~mm}$.

Next we compare the results obtained in the present experiment with the results reported in Refs. [13,17]. Compared to Ref. [13], the first demonstration of the beam focusing with the help of the crystal produced on the basis of strip crystal [16] technology has been reported in [17]. Although the focusing effect was observed in the experiment, some imperfections were found in the samples studied. Primarily these are the nonuniformity of the crystal bending and a large angular spread of the beam downstream of the crystal deflector (three times larger than the critical angle).

As the results from the present paper demonstrate, our improved method of fabrication achieved important improvements, namely: (1) a very good linearity of deflection angle as a function of the transverse coordinate; (2) the angular spread was reduced to about the critical angle $\theta_{c} ;(3)$ the mean deflection angle of proton (pion) beams was increased by a factor $\sim 10-20$; (4) the transverse thickness of crystal deflectors was substantially increased (up to $2-4 \mathrm{~mm}$ ).
In particular, due to realization of items (1) and (2), the reduction factor [see Eq. (13)] increased by a factor 2-2.5 compared with previous results [17].

The influence of the angular characteristics of channeled particles on the parameters of beam focusing was investigated for the first time. The important result is that these parameters are practically independent of the maximum entrance angle $\phi$.

\section{CONCLUSIONS}

Four focusing crystals were fabricated and tested in a $400 \mathrm{GeV} / c$ proton beam and $180 \mathrm{GeV} / c$ secondary beams at the $\mathrm{H} 8$ beam line of the CERN SPS. In manufacturing crystal deflectors, we have profited from previous experience and have shown that, in addition to beam focusing, these crystal devices could deflect the full beam through a significant angle. We have evaluated these crystals in both the focusing and defocusing modes.

The tests have been performed with the help of two methods of reconstruction of the beam envelope. The first is based on common relations for particle motion and is independent of the form of the crystal cut. The second method assumes a linear crystal cut. The results obtained using these methods yield similar results for the characteristics of the beam envelope. This demonstrates the high fabrication quality of the focusing devices and in particular the excellent linearity of cutting of the crystal edge.

Focusing (defocusing) crystals may be considered as useful tools for steering beams, for example in collimation, in obtaining secondary beams and for other applications.

It is important that the quality of focusing by crystalbased devices increases with the growth of the beam energy. In this way, new possibilities open for these devices for manipulation of ultra-high energy beams.

Summarizing, the progress achieved after the results of [17] had been published is as follows: (a) the quality of focusing crystals has been improved; (b) the defocusing of the beam by the crystal deflectors has been studied; (c) the impact of various factors such as $\left\langle x^{2}\right\rangle-\bar{x}^{2}, k / R$ and $\left\langle\theta^{2}\right\rangle-\bar{\theta}^{2}$ parameters on the characteristics of focusing necessary for designing focusing devices for high energy particle beams has been understood.

\section{ACKNOWLEDGMENTS}

The IHEP participants in the UA9 experiment acknowledge financial support from the Russian Science Foundation (Grant No. 17-12-01532). The Imperial College group thanks the UK Science and Technology Facilities Council for financial support. The INFN authors acknowledge the support of the ERC Ideas Consolidator Grant No. 615089 CRYSBEAM. 
[1] E. N. Tsyganov, Report No. FERMILAB-TM-0682, Batavia, 1976.

[2] A. Asseev, M. Bavizhev, E. Ludmirsky, V. Maisheev, and Y. Fedotov, Extraction of the $70 \mathrm{Gev}$ proton beam from the IHEP accelerator towards beam line 2(14) with a bent single crystal, Nucl. Instrum. Methods Phys. Res., Sect. A 309, 1 (1991).

[3] H. Akbari, First results on proton extraction from the cernsps with a bent crystal, Phys. Lett. B 313, 491 (1993).

[4] C. T. Murphy et al., First results from bent crystal extraction at the Fermilab Tevatron, Nucl. Instrum. Methods 119, 231 (1996).

[5] W. Scandale, Observation of channeling for $6500 \mathrm{Gev} / \mathrm{c}$ protons in the crystal assisted collimation setup for $\mathrm{fLHCg}$, Phys. Lett. B 758, 129 (2016).

[6] V. G. Baryshevsky, Spin rotation and depolarization of relativistic particles traveling through a crystal, Nucl. Instrum. Meth. B 44, 266 (1990).

[7] A. V. Khanzadeev, V. M. Samsonov, R. A. Carrigan, and D. Chen, Experiment to observe the spin precession of channeled relativistic $\Sigma^{+}$hyperons, Nucl. Instrum. Methods 119, 266 (1996).

[8] A. M. Taratin and S. A. Vorobiev, Volume reflection of high-energy charged particles in quasi-channeling states in bent crystals, Phys. Lett. A 119, 425 (1987).

[9] W. Scandale, A. Vomiero, S. Bacordi et al., Volume Reflection Dependence of $400 \mathrm{GeV} / \mathrm{c}$ Protons on the Bent Crystal Curvature, Phys. Rev. Lett. 101, 234801 (2008).

[10] N. Mokhov, J. Annala, R. Carrigan et al., Tevatron beam halo collimation system: design, operational experience and new methods, J. Instrum. 6, T08005 (2011).

[11] Yu. A. Chesnokov, V. I. Kotov, V. A. Maisheev, and I. A. Yazynin, Radiation of photons in process of charged particle volume reflection in bent monocrystal, J. Instrum. 3, P02005 (2008).

[12] W. Scandale et al., Experimental study of the radiation emitted by $180-\mathrm{GeV} / \mathrm{c}$ electrons and positrons volumereflected in a bent crystal, Phys. Rev. A 79, 012903 (2009).

[13] M. A. Gordeeva, M. P. Gur'ev, A. S. Denisov et al., First results on the focusing of a $70-\mathrm{GeV}$ proton beam by a curved single crystal, JETP Lett. 54, 487 (1991).
[14] V. I. Baranov, V. M. Biryukov, A. P. Bugorsky, Yu. A. Chesnokov, V. I. Kotov, M. V. Tarakanov, V. I. Terekhov, S. V. Tsarik, O. L. Fedin, M. A. Gordeeva, M. P. Gur'ev, Yu. P. Platonov, and A.I. Smirnov, Highly efficient deflection of a divergent beam by a bent single crystal, Nucl. Instrum. Methods Phys. Res., Sect. B 95, 449 (1995).

[15] A. G. Afonin et al., A device based on a bent crystal with a variable curvature for controlling particle beams at accelerators, Instrum. Exp. Tech. 56, 123 (2013).

[16] A. G. Afonin, V. M. Biryukov, V. A. Gavrilushkin et al., First results of experiments on high-efficiency singlecrystal extraction of protons from the U-70 accelerator, J. Exp. Theor. Phys. Lett. 67, 781 (1998).

[17] W. Scandale et al., Observation of focusing of $400 \mathrm{GeV} / \mathrm{c}$ proton beam with the help of bent crystals, Phys. Lett. B 733, 366 (2014); 734, 408 (2014).

[18] V. M. Biryukov, Yu. A. Chesnokov, and V. I. Kotov, Crystal channeling and its application at high-energy accelerators (Springer, Berlin, 1997), p. 219.

[19] V. A. Maisheev, Yu. A. Chesnokov, and P. N. Chirkov, Focusing of high energy particles with the help of bent single crystal, Nucl. Instrum. Methods Phys. Res., Sect. B 355, 360 (2015).

[20] M. Pesaresi, W. Ferguson, J. Fulcher, G. Hall, M. Raymond, M. Ryan, and O. Zorba, Design and performance of a high rate, high angular resolution beam telescope used for crystal channeling studies, J. Instrum. 6, P04006 (2011).

[21] E. Bagli, V. Guidi, and V. Maisheev, Calculation of the potential for interaction of particles with complex atomic structures, Phys. Rev. E 81, 026708 (2010).

[22] W. Scandale et al., Observation of nuclear dechanneling for high-energy protons in crystals, Phys. Lett. B 680, 129 (2009).

[23] V. A. Maisheev and Y. A. Chesnokov, New beam optics on the basis of bent single crystals, Nucl. Instrum. Methods Phys. Res., Sect. B 402, 300 (2017).

[24] A. G. Afonin et al., Pis'ma Zh. Eksp. Teor. Fiz. 104, 9 (2016) [Focusing crystal device for deflecting a divergent 50-GeV proton beam, JETP Lett. 104, 12 (2016)]. 